\title{
Epigenetics in Dentistry: A Literature Review
}

\section{Abstract}

Ongoing research is continuously discovering the role of epigenetics in various human diseases and disorders. If every single cause and effect of the different combinations of genes could be mapped, and if the gene's state could be reversed to keep the good while eliminating the bad, then epigenetics could cure cancer, slow aging, stop obesity; prevent and treat dental problems. Dental field can greatly benefit from latest research in this area. There could be range of opportunities for diagnosis, treatment and prevention. Potential research of epigenetics for oral health includes the inflammation and immune responses that causes periodontitis and the development of oral malignancies. There is possibility to doze off body's response to oral disease to prevent loss of teeth and protect dental implants.

Keywords: Epigenetics; Dentistry; DNA; Genes; Prevention; Future treatment

Received: December 12, 2016; Accepted: January 03, 2017; Published: January 09, 2017

\section{Epigenetics}

Epigenetics is the study of changes in gene function that are mitotically and/or meiotically heritable and that do not entail a change in DNA sequence

Epigenetic change is a regular and natural occurrence but can also be influenced by several factors including age, environment and disease state. Epigenetic modifications can be seen as commonly as the manner in which skin cells, liver cells, brain cells differentiates. Epigenetic change can have more damaging effects that can result in diseases like malignancies. DNA methylation, Histone modification and Non-coding RNA-associated gene silencing are three systems currently considered to initiate and sustain epigenetic change [1]. Ongoing research is continuously discovering the role of epigenetics in a various human diseases and disorders. Epigenetic research involved human subjects; studies in dentistry included periodontium and dental pulp cells.

\section{History of epigenetics}

Waddington during the mid-twentieth century have evolved into the field of epigenetics. The term epigenetics, which was coined by Waddington in 1942, was derived from the Greek word "epigenesis" which originally described the influence of genetic processes on development [2]. During the 1990s there became a renewed interest in genetic assimilation. This lead to elucidation of the molecular basis of Conrad Waddington's observations in which environmental stress caused genetic assimilation of certain phenotypic characteristics in Drosophila fruit flies. Since then, research efforts have been focused on unraveling the epigenetic

\section{Abdul Habeeb Bin Mohsin ${ }^{1}$ and Sheraz Barshaik²}

\author{
1 Department of Prosthodontics, Sri Sai \\ College of Dental Surgery, Vikarabad, \\ India
}

2 Department of Oral and Maxillofacial Surgery, MNR Dental College and Hospital, Sangareddy, India

\section{Corresponding author:}

Abdul Habeeb Bin Mohsin

\section{doc.habeeb@gmail.com}

Department of Prosthodontics, Sri Sai College of Dental Surgery, Vikarabad, Hyderabad, 501101, India.

Tel: +919849990569

Citation: Mohsin AHB, Barshaik S. Epigenetics in Dentistry: A Literature Review. J Clin Epigenet. 2017, 3:1.

mechanisms related to these types of changes [3].

Currently, DNA methylation is one of the most broadly studied and well-characterized epigenetic modifications dating back to studies done by Griffith and Mahler in 1969 which suggested that DNA methylation may be important in long term memory function [4]. Other major modifications include chromatin remodeling, histone modifications, and non-coding RNA mechanisms. The renewed interest in epigenetics has led to new findings about the relationship between epigenetic changes and a host of disorders including various cancers, mental retardation associated disorders, immune disorders, neuropsychiatric disorders and pediatric disorders.

\section{Epigenetics in Dentistry}

Review of Literature: Gomez et al. conducted a review to show evidence that supports the hypothesis that epigenetic alteration, such as hyper and hypomethylation of cytokine genes could help to understand the mechanisms related to periodontal disease activity [5]. Therefore, epigenetics may have future impact on diagnosis and/or therapeutics of periodontal disease.

Loo et al. [6] confirmed that E-cadherin and COX-2 expression are 
factors related to periodontitis. Further study of similar epigenetic changes may prove to be extremely useful in the diagnosis and treatment of chronic periodontitis in the future.

Dayarsaihan [7] stated that progress in research of epigenetics in inflammatory response opens opportunities for the development of efficient medications for particular therapies. Some of the drugs proposed for the therapy are histone deacetylase inhibitors and de-methylating agents. Proposed drugs targets dividing tumor cells and restore normal cell functions. The latest technological achievements will be instrumental in the development of epigenetic drugs with greater specificity.

SD Williams stated that epigenetics is proving to be a valuable and insightful arm of genomics research [8]. Whilst there are no practical applications in dentistry at present, epigenetics may have profound influences in the future and so all clinicians should be aware of its basic principles. Even though it is early days, Australian periodontal research is demonstrating that epigenetic therapy might one day be an effective treatment for periodontitis. Furthermore, in terms of dental development; it may be possible to intervene early on to prevent hypodontia and a range of dental anomalies. In the shorter time frame though, epigenetics could be used as a reliable screening tool for a range of dental anomalies, including inherited enamel defects, as well as a means of assessing an individual's susceptibility to dental caries and periodontal disease. Exciting times lie ahead!

Seo et al. enlightened relation between epigenetic changes and periodontal diseases [9]. They also enlightened the epigenetic association with inflamed dental pulp cells. They said more research is required to support these initial studies through which better understanding of the effect of epigenetics on dental health and development of effective therapies may be established.

Fernando et al. has outlined potential evidence on the interrelationship of epigenetics with social and environmental predictors for dental caries in child population [10]. From the evidence they expected that appropriate interventions will be recognized and implemented to tackle dental caries in children and improve oral health and quality of life of the affected. However, obtaining a larger sample size was one limitation of the study.

Ari et al. stated the prevalence of the periodontal disease is higher in India [11]. They highlighted that DNA methylation and histone modifications occur in the oral mucosa in response to bacteria and inflammatory process. Hence, identifying the genetic factors and epigenetic variations in periodontitis will be useful in developing innovative therapeutic interventions.

\section{Periodontics}

When body responds to infection, the resulting inflammation and other immune responses can contribute to bone loss and ligament damage that leads to tooth loss. Currently, specific bacteria can be detected in mouth, and tell whether it has genetic tendency toward serious periodontal disease, but we may also be able to determine whether body is triggering a negative response [8]. The epigenetic changes on pro-inflammatory mediators in periodontal disease have been linked to a number of environmental stimuli, including smoking and nutrition, and the oral bacteria themselves. lacopino stated that these changes in the host tissues can facilitate bacterial colonization, increase inflammatory damage and also provide bacteria with increased levels of carbohydrate for metabolism [12]. He also noted that these findings have implications for the methods used to diagnose periodontal disease and to identify patients at risk. He suggested that a new approach to management of periodontal problems in the future, based on personalized medicine, is likely to consider additional factors apart from bleeding and pocket depths, including types of bacteria present in the biofilm and epigenetic changes in the periodontal tissues [12].

\section{Craniofacial Development}

Carlson has reviewed the various theories and concepts of craniofacial growth \& development \& related them to the developments in the field of genetics [13]. In discussing developments in the post-genomic era, he noted that there is an increasing awareness of the genes and their products that regulate craniofacial development, including the notion that these genes are turned on and off at critical times. He stated that the issue is not the fact that intrinsic factors within the genome regulate morphogenesis, but that the complex interaction of cells and tissues with remote extrinsic factors within the body and the environment are triggers, or switches for gene expression that influences postnatal growth and responsiveness to clinical treatment. Carlson goes on to speculate that within the next several decades, orthodontists will be using diagnostic molecular kits for growth-related problems and to determine precisely each patient's developmental status as well as the presence or absence of abnormal response [8].

\section{Oral Malformations}

For such complex and heterogeneous disorders, a multifactorial model of inheritance is favored in which genetic risk factors interact with environmental co- variates [14]. In a genome-wide study when gene-environment models were applied to three common environmental exposures in pregnancy, the significant interactions were found with alcohol consumption, maternal smoking and multivitamin supplementation $[15,16]$. Future studies will further elucidate the role of epigenetic factors in cleft lip/cleft palate and provide a basis for sound preventive advice to reduce the frequency of this distressing condition; the dental profession will have a role in providing this advice.

\section{Tooth Development and Defects}

The clinical aspects of this topic are covered in a study by Seow [17] noted the influence of DNA methylation on the enamel protein, (amelogenin), produced from genes on the $X$ and $Y$ chromosomes.

\section{Implant dentistry}

Based on the several studies, advance epigenetics will be able to turn on or off the genes responsible for body's negative response. Epigenetic treatment may be able to turn off body's response to periodontal disease to give time for treatments and antibiotics to work. This could protect dental implants [8]. 


\section{Future of Epigenetics in Dentistry}

Dentistry can greatly benefit from new research in this area. It could open up a range of opportunities for diagnosis, treatment and prevention. It is known that genome plays an important role in tooth development and in a range of oral diseases; the oral microbiota also play a key role in the state of oral health. Presently there is a potential to develop an epigenetic profile of a patient and provide a more personalized level of care.
Potential research of epigenetics for oral health includes the inflammation and immune responses that causes periodontitis and the development of oral malignancies. There are possibilities to doze off body's response to oral disease to prevent loss of teeth and protect dental implants. Most importantly we can conduct screening for many of these potential oral health problems from an early age so that it can be prevented or its impact can be reduced. Epigenetics has much to offer in the future treatment and prevention of dental disease. 


\section{References}

1 Egger G, Liang G, Aparicio A, Jones PA (2004) Epigenetics in human disease and prospects for epigenetic therapy. Nature 429: 457-463.

2 Waddington $\mathrm{CH}$ (1942) “The epigenotype". Endeavour 1: 18-20.

3 Brouwer JR (2012) A Crash Course in Epigenetics Part 1: An intro to epigenetics. Bitesize Bio.

4 Holliday R (2006) Epigenetics: A Historical Overview. Epigenetics 1: 76-80.

5 Gomez RS, Dutra WO, Moreira PR (2009) Epigenetics and periodontal disease: future perspectives. Inflamm Res 8: 625-629.

6 Loo WTY, Jin L, Mary NB, Wang CM, Chow LWC (2010) Epigenetic change in E-cardherin and COX-2 to predict chronic periodontitis. J Transl Med 8: 110.

7 Dayarsaihan D (2011) Epigenetic Mechanisms in Inflammation. J Dent Res 90: 9-17.

8 Williams SD, Hughes TE, Adler CJ, Brook AH, Townsend GC (2014) Epigenetics: A New Frontier in Dentistry. Aust Dent J 5: 23-33.

9 Seo JY, Park YJ, Yi YA, Hwang JY, Lee IB, et al. (2015) Epigenetics: general characteristics and implications for oral health. Restor Dent Endod 40: 14-22.
10 Fernando S, Speicher DJ, Bakr MM, Benton MC, Lea RA, et al. (2015) Protocol for assessing maternal, environmental and epigenetic risk factors for dental caries in children. BMC Oral Health 15: 167.

11 Ari G, Cherukuri S, Namasivayam A (2016) Role of Epigenetic Alterations in Periodontitis. J Clin Diagn Res 10: ZE07-ZE09.

12 lacopino AM (2010) Epigenetics: new explanations for old problems? J Can Dent Assoc 76: a76.

13 Carlson DS (2005) Theories of craniofacial growth in the postgenomic era. Semin Orthod 11: 172-83.

14 Dixon MJ, Marajita ML, Beaty TH, Murray JC (2011) Cleft lip and palate: understanding genetic and environmental influences. Nat Rev Genet 12: 167-178.

15 Marazita ML (2012) The evolution of human genetic studies of cleft lip and cleft palate. Annu Rev Genome Human Genet 3: 263-283.

16 Beaty TH, Ruczinski I, Murray JC, Marazita ML, Munger RG, et al. (2011) Evidence for gene environment interaction in a genome wide study of nonsyndromic cleft palate. Genet Epidemiol 35: 469-478.

17 Seow WK (2014) Developmental defects of enamel and dentine: challenges for basic science research and clinical management. Aust Dent J 59: 143-154. 